\title{
The Assessment of Risk for Gastrointestinal Injury with Anticoagulant and Antiplatelet Drugs: The Possible Beneficial Effect of Eicosapentaenoic Acid for the Risk of Gastrointestinal Injury
}

\author{
Mamoru Tanaka, ${ }^{* a}$ Akihiro Tanaka, ${ }^{a}$ Katsuya Suemaru, ${ }^{b}$ and Hiroaki Araki ${ }^{a}$ \\ ${ }^{a}$ Division of Pharmacy, Ehime University Hospital; Shitsukawa, Toon, Ehime 791-0295, Japan: and ${ }^{b}$ School of \\ Pharmacy, Shujitsu University; 1-6-1 Nishigawara, Naka, Okayama 703-8516, Japan. \\ Received July 3, 2012; accepted November 25, 2012; advance publication released online December 3, 2012
}

\begin{abstract}
Antithrombotic drugs have been increasingly used for treating ischemic cardiovascular diseases among the elderly in Japan. However, antithrombotic drugs are known to be risk factors for gastrointestinal injury. Therefore, we conducted a pharmacoepidemiologic study on patients receiving antithrombotic drugs to identify the risk factors for gastrointestinal injury. This retrospective case-control study included patients who were prescribed antithrombotic drugs at the Ehime University Hospital between April and September 2010. Of the 3271 patients who received antithrombotic drug therapy, 172 (5.3\%) developed gastrointestinal injuries, including gastric ulcers, duodenal ulcers, and hemorrhagic gastrointestinal injuries. Further, the incidence of gastrointestinal injury was higher in patients with hypertension than in those without $(p<0.0001)$. Multivariate adjusted odds ratios and $\mathbf{9 5 \%}$ confidence intervals were calculated using stepwise logistic regression. The adjusted odds ratios for gastrointestinal injury were 1.56 (95\% confidence interval 1.07-2.36) for hypertension, $1.70(1.17-2.50)$ for low-dose aspirin, 2.77 (1.70-4.49) for clopidogrel, 1.95 (1.23-3.08) for warfarin, and $4.13(2.88-5.95)$ for nonsteroidal anti-inflammatory drugs. On the other hand, the nonadjusted odds ratio for drug-associated gastrointestinal injury was $0.43(0.20-0.84)$ for eicosapentaenoic acid (EPA). In addition, we found that patients aged 70 years or older were at increased risk of drug-associated gastrointestinal injury. These findings suggest that while many antithrombotic drugs are risk factors for gastrointestinal injury, EPA may be a safe option for suppressing or preventing gastrointestinal injury.
\end{abstract}

Key words antithrombotic drug; gastrointestinal injury; eicosapentaenoic acid; nonsteroidal anti-inflammatory drug; elderly patient

At present, people older than 65 years account for more than $22 \%$ of the entire population in Japan, satisfying the United Nations definition of "ultra-ageing society" (percentage of elderly people, over $21 \%$ ). Elderly people tend to have multiple comorbidities. Myocardial infarction and cerebral infarction are representative geriatric diseases whose incidence increases with age. In Japan, these ischemic cardiovascular diseases are major causes of death. Cerebral infarction is a disease causing serious problems related to the welfare of people since they are likely to develop sequelae (hemiplegia), making them dependent on the assistance from others during daily life.

When the "Guidelines on Anticoagulant and Antiplatelet Drug Therapy for Cardiovascular Diseases" were revised in 2009, the management of gastrointestinal ulcers caused by antiplatelet drugs in clinical practice was added as another important topic. ${ }^{1)}$ This revision was based on the fact that while the onset of peptic ulcers had decreased as a result of Helicobacter pylori eradication therapy, there had been an elevation in the risk of gastrointestinal complications (gastrointestinal ulcers or hemorrhagic gastrointestinal lesions) associated with nonsteroidal anti-inflammatory drugs (NSAIDs) and antiplatelet/anticoagulant drugs (antithrombotic drugs). Sakamoto et al. conducted a case-control study in patients with gastrointestinal bleeding, reporting that the risk of hemorrhagic events rose 5.5- to 7.7-fold in patients taking aspirin. ${ }^{2)}$

The above-mentioned guidelines on anticoagulant and antiplatelet drug therapy recommend measures for the prevention of gastrointestinal complications in high-risk patients

The authors declare no conflict of interest. requiring aspirin treatment. It has been reported that histamine type 2-receptor antagonists (H2RAs) and proton pump inhibitors (PPIs) were effective in the prevention of gastroduodenal ulcers induced by low-dose aspirin. ${ }^{3)}$ To date, however, few reports on the relationship between the type of antithrombotic drugs and gastrointestinal injury have been published in Japan. ${ }^{3,4)}$ Reducing the risk of gastrointestinal injury (e.g., gastrointestinal ulcers and gastrointestinal bleeding) is also an important clinical issue from the viewpoint of facilitating continued treatment with antithrombotic drugs. With these points borne in mind, it is essential to prevent and treat thrombotic cardiovascular and cerebrovascular injuries and to select appropriate antithrombotic drugs. ${ }^{2-7)}$ However, there are many unresolved questions about the risk of gastrointestinal injury arising from antithrombotic drugs other than low-dose aspirin and the effects of drugs used for the prevention of such injury. Therefore, we carried out a pharmacological study in patients receiving antithrombotic drugs in order to identify the risk factors of gastrointestinal injury associated with antithrombotic drug therapy.

\section{MATERIALS AND METHODS}

Subjects and Methods A case-control analysis was carried out, involving patients for whom antithrombotic drugs were prescribed at the Ehime University Hospital (hereinafter called "our hospital"). Cases (patients having developed gastrointestinal injury) were randomly matched with controls (age- and gender-matched patients free of gastrointestinal injury) at a ratio of $1: 4$. The risk of gastrointestinal injury was classified according to the presence-absence of injury. 
Univariate logistic regression analysis was carried out to determine which drugs and life-style related diseases served as significant factors. On each factor identified by univariate logistic regression analysis as a significant factor, multivariate logistic regression analysis was carried out to identify risk factors of gastrointestinal injury. Furthermore, to analyze the association of risk factors with aging, multivariate logistic regression analysis was carried out by stratifying patients aged over 50 years by age at intervals of 10 years. The survey on patients having developed a gastrointestinal injury was carried out on those patients for whom antithrombotic drugs were prescribed for more than $7 \mathrm{~d}$ at our hospital between April and September 2010. A retrospective survey was conducted on the healthcare data contained in the medical records, including gender, age, drug (antithrombotic drugs, NSAIDs), gastrointestinal injury, and habit-related disease. This study was carried out in accordance with the Ethical Guidelines on Epidemiological Research (Ministry of Education, Culture, Sports, Science and Technology and Ministry of Health, Labour and Welfare) under approval of the Ethics Committee of the Ehime University School of Medicine.

Drugs The antithrombotic drugs investigated were as follows: (1) drugs with antiplatelet activity, i.e., aspirin $(100 \mathrm{mg})$; combination preparation of aspirin $(81 \mathrm{mg})$ and dialuminate $(33 \mathrm{mg})$; ticlopidine hydrochloride; cilostazol; eicosapentaenoic acid (EPA; an $\omega-3$ polyunsaturated fatty acid); beraprost sodium; sarpogrelate hydrochloride; clopidogrel bisulfate; limaprost; dipyridamole; dilazep hydrochloride; trapidil; ibudilast; and ifenprodil tartrate, (2) a drug with anticoagulant activity, i.e., warfarin potassium, and (3)NSAIDs used in our hospital, which are known to induce gastrointestinal injury frequently, i.e., mefenamic acid, ibuprofen, naproxen, flurbiprofen, loxoprofen sodium, indomethacin, indomethacin farnesil, etodolac, diclofenac sodium, nabumetone, meloxicam, lornoxicam, and celecoxib.

Gastrointestinal Injury Gastrointestinal injury was judged to be "present" in cases where any of the antithrombotic drugs were used during the survey period and a definite diagnosis of any of the following diseases according to the International Classification Diseases 10th Revision (ICD10) classification was made during the survey period: K25.0-9 (gastric ulcers), K26.0-9 (duodenal ulcers), K27.9 (peptic ulcers of unidentified site), K28.9 (gastrojejunal ulcers), K29.0 (acute hemorrhagic gastritis), K92.1 (lower gastrointestinal bleeding), and K92.2 (gastrointestinal bleeding, gastric ulcers with unknown details). K29.1-9 (gastritis and duodenitis), K21.0-9 (gastroesophageal regurgitation), and T81.0 (bleeding and hematoma secondary to intervention, not classified in any other category) were excluded. Because of the limitations imposed on this study, cases with relapsing gastrointestinal injury, presence/absence of $H$. pylori infection, and history of treatment were not taken into consideration.

Life-Style Related Diseases Diabetes mellitus, hypertension, and dyslipidemia (hyperlipidemia) were selected as lifestyle related diseases serving as risk factors for thrombotic cerebellar-cardiovascular diseases.

Statistical Analysis All analyses were made using JMP ${ }^{\circledR}$ software 8.0.2 (SAS Institute Inc., Cary, NC, U.S.A.). Fisher's exact test or Student's $t$-test was employed for comparison between 2 groups. In all analyses, $p<0.05$ (two-tailed) was regarded statistically significant. Analysis of risk factors was carried out by univariate logistic regression analysis and stepwise multivariate logistic regression analysis to determine the odds ratio (OR), adjusted odds ratio (AOR), and 95\% confidence interval $(95 \% \mathrm{CI})$.

\section{RESULTS}

Survey of Patients Treated with Antithrombotic Drugs During the survey period, 3271 patients received treatment with antithrombotic drugs and 172 patients (5.3\%) developed gastrointestinal injury. The type of gastrointestinal injury among the 172 patients was gastric ulcer in 122 cases, duodenal ulcer in 3 cases, and hemorrhagic gastrointestinal injury in 47 cases (Table 1). There was no significant difference in the onset of gastrointestinal injury, except for the age at onset,

Table 1. Classification of Gastrointestinal Injury

\begin{tabular}{|c|c|c|c|}
\hline Classification & No. of patients & Disease & No. of patients \\
\hline \multirow{2}{*}{$\begin{array}{l}\text { Nonhemor- } \\
\text { rhagic }\end{array}$} & \multirow[t]{2}{*}{125} & Gastric ulcer & 122 \\
\hline & & Duodenal ulcer & 3 \\
\hline \multirow[t]{5}{*}{ Hemorrhagic } & \multirow[t]{5}{*}{47} & $\begin{array}{l}\text { Hemorrhagic } \\
\text { gastric ulcer }\end{array}$ & 19 \\
\hline & & $\begin{array}{l}\text { Hemorrhagic } \\
\text { duodenal } \\
\text { ulcer }\end{array}$ & 3 \\
\hline & & $\begin{array}{c}\text { Gastrointestinal } \\
\text { hemorrhage }\end{array}$ & 7 \\
\hline & & $\begin{array}{l}\text { Upper gastro- } \\
\text { intestinal } \\
\text { hemorrhage }\end{array}$ & 17 \\
\hline & & $\begin{array}{l}\text { Lower gastro- } \\
\text { intestinal } \\
\text { hemorrhage }\end{array}$ & 1 \\
\hline
\end{tabular}

Table 2. Comparison of Characteristics of Patients with and without Gastrointestinal Injury

\begin{tabular}{|c|c|c|c|c|c|c|}
\hline \multirow{3}{*}{ Variable } & \multirow{3}{*}{ Total No. of patients } & \multicolumn{4}{|c|}{ Gastrointestinal injury } & \multirow{3}{*}{$p$ Value } \\
\hline & & \multicolumn{2}{|c|}{ Injury } & \multicolumn{2}{|c|}{ No injury } & \\
\hline & & No. of patients & $\%$ & No. of patients & $\%$ & \\
\hline Total & $3271(\%)$ & 172 & 5.3 & 3099 & 94.7 & \\
\hline Sex & & & & & & $\left.0.5820^{a}\right)$ \\
\hline Male & $1790(54.7)$ & 98 & 57.0 & 1692 & 54.6 & \\
\hline Female & $1481(45.3)$ & 74 & 43.0 & 1407 & 45.4 & \\
\hline \multicolumn{7}{|l|}{ Age (year) } \\
\hline Mean \pm S.D. & $69.2 \pm 12.8$ & $70.0 \pm 14.0$ & & $66.2 \pm 18.0$ & & $0.0056^{b)}$ \\
\hline
\end{tabular}

a) Fischer's test, $b$ ) $t$-test, S.D.: standard deviation. 
which was significantly higher in patients developing gastrointestinal injury $(p<0.01$, Table 2$)$.

Life-Style Related Diseases and Gastrointestinal Injury The incidence of gastrointestinal injuries in patients with diabetes mellitus, hypertension, or dyslipidemia is shown in Table 3. Hypertensive patients receiving antithrombotic drugs developed gastrointestinal injury at an incidence of $7.2 \%$, which was significantly higher than that of nonhypertensive patients $(p<0.0001)$.

Case-Control Study A case-control study was carried out, involving 172 cases of gastrointestinal injury and 688 gender- and age-matched controls. The control subjects were selected from 3099 patients who did not have gastrointestinal injury and who received antithrombotic-anticoagulant drugs. There was no significant difference in the male-to-female ratio or age between the case group and the control group (Table 4).

Risk Factors of Gastrointestinal Injury (Univariate Logistic Regression Analysis) The data on the OR for gastrointestinal injury of 14 antithrombotic drugs are summarized in Table 5. The unadjusted OR (95\%CI, $p$ value) calculated by univariate logistic regression analysis in the case-control study was $2.95(1.90-4.56, p<0.0001)$ for clopidogrel, $1.77(1.26-2.49, p=0.0010)$ for low-dose aspirin, and $1.77(1.18-2.61, p=0.0048)$ for warfarin potassium; for each drug, the OR was significantly higher than that for the control group. The unadjusted OR for NSAIDs was 4.20 (2.97-5.97, $p<0.001$ ), which was also significantly higher than that of the control group similarly to 3 antithrombotic drugs. The unadjusted OR for EPA was $0.43(0.20-0.84, p=0.0207)$, significantly lower than 1 (Table 5).

Independent Risk Factors of Gastrointestinal Injury (Multivariate Logistic Regression Analysis) Factors found to be significantly associated with gastrointestinal injury in the univariate logistic regression analysis were subjected to multivariate logistic regression analysis and the adjusted odds ratio $(95 \% \mathrm{CI})$ was calculated. This analysis revealed the following independent risk factors: hypertension, 1.56 (1.07-2.36, $p=0.0242)$; low-dose aspirin, $1.70(1.17-2.50, \quad p=0.0061)$;

Table 3. Classification of Diseases and Occurrence of Gastrointestinal Injury

\begin{tabular}{ccccr}
\hline \hline & No. of patients & Occurrence of injury & Frequency (\%) & $p$ Value \\
\hline Diabetes & 1795 & 106 & 5.9 & 0.3032 \\
Hypertension & 1716 & 123 & 7.2 & $<0.0001$ \\
Dyslipidemia & 1191 & 70 & 5.9 & 0.5196 \\
\hline
\end{tabular}

Fischer's test.

Table 4. Comparison of Characteristics of Patients with Gastrointestinal Injury and Matched Controls without Injury

\begin{tabular}{|c|c|c|c|c|c|c|}
\hline \multirow{3}{*}{ Variable } & \multirow{3}{*}{ Total No. of patients } & \multicolumn{4}{|c|}{ Gastrointestinal injury } & \multirow{3}{*}{$p$ Value } \\
\hline & & \multicolumn{2}{|c|}{ Injury } & \multicolumn{2}{|c|}{ No injury } & \\
\hline & & No. of patients & $\%$ & No. of patients & $\%$ & \\
\hline Total & $860(\%)$ & 172 & 20.0 & 688 & 80.0 & \\
\hline Sex & & & & & & $0.9315^{a)}$ \\
\hline Male & $493(57.3)$ & 98 & 57.0 & 395 & 57.4 & \\
\hline Female & 367 (42.7) & 74 & 43.0 & 293 & 42.6 & \\
\hline Age (year) & & & & & & \\
\hline Mean \pm S.D. & $70.0 \pm 13.6$ & $70.0 \pm 14.0$ & & $70.0 \pm 13.5$ & & $0.9940^{b)}$ \\
\hline
\end{tabular}

a) Fischer's test, $b$ ) $t$-test, SD: standard deviation.

Table 5. Risk Factors of Gastrointestinal Injury (Odds Ratio)

\begin{tabular}{|c|c|c|c|c|c|}
\hline & No. of patients & Occurrence of injury & Odds ratio & $95 \% \mathrm{CI}$ & $p$ Value \\
\hline LDA & 413 & 102 & 1.77 & $1.26-2.49$ & 0.0010 \\
\hline Ticlopidine hydrochloride & 60 & 8 & 0.60 & $0.26-1.21$ & 0.1851 \\
\hline Cilostazol & 55 & 14 & 1.40 & $0.72-2.57$ & 0.2979 \\
\hline EPA & 87 & 9 & 0.43 & $0.20-0.84$ & 0.0207 \\
\hline Beraprost sodium & 57 & 9 & 0.74 & $0.33-1.47$ & 0.4125 \\
\hline Sarpogrelate & 39 & 5 & 0.58 & $0.20-1.37$ & 0.2569 \\
\hline Clopidogrel & 104 & 40 & 2.95 & $1.90-4.56$ & $<0.0001$ \\
\hline Limaprost alfadex & 71 & 9 & 0.56 & $0.25-1.09$ & 0.1117 \\
\hline Dipyridamole & 10 & 2 & 1.00 & $0.15-4.03$ & 1.000 \\
\hline Dilazep hydrochloride hydrate & 4 & 1 & 1.34 & $0.07-10.50$ & 0.8028 \\
\hline Trapidil & 1 & 0 & - & - & - \\
\hline Ibudilast & 23 & 1 & 0.18 & $0.01-0.85$ & 0.0916 \\
\hline Ifenprodil tartrate & 44 & 5 & 0.50 & $0.17-1.17$ & 0.1491 \\
\hline Warfarin potassium & 160 & 45 & 1.77 & $1.18-2.61$ & 0.0048 \\
\hline NSAIDs & 255 & 96 & 4.20 & $2.97-5.97$ & $<0.0001$ \\
\hline
\end{tabular}

CI: confidence interval, LDA: low-dose aspirin, EPA: eicosapentaenoic acid, NSAIDs: nonsteroidal anti-inflammatory drugs. Fischer's test. 
clopidogrel, $2.77(1.70-4.49, p<0.0001)$; warfarin potassium, $1.95(1.23-3.08, p=0.0041) ;$ and NSAIDs, 4.13 (2.88-5.95, $p<0.0001$ ) (Table 6).

Risk Factors of Gastrointestinal Injury Analyzed by Age To evaluate the influence of age on gastrointestinal injury, the 792 patients aged over 50 years were stratified by age (at intervals of 10 years), and the independent risk factors of gastrointestinal injury (Table 6) were analyzed by age (multivariate logistic regression analysis). In patients aged 70 years or older, the following independent risk factors were identified: hypertension, $2.02(1.19-3.56, p=0.00116)$; low-dose aspirin, 1.91 (1.17-3.16, $p=0.0103)$; clopidogrel, 3.07 (1.62-5.57, $p=0.0005)$; warfarin potassium, $2.45(1.35-4.43, p=0.0030)$; and NSAIDs, 4.26 (2.65-6.93, $p<0.0001)$. NSAIDs were also identified as an independent risk factor in each age group over 50 years and clopidogrel was an independent risk factor in the age group of 60 to 69 years (Table 7).

\section{DISCUSSION}

We conducted an investigation on patients receiving antithrombotic drugs to analyze the risk of developing gastrointestinal injury on taking antithrombotic drugs. The analysis of gastrointestinal injury in relation to life-style related diseases revealed that the percentage of patients developing gastrointestinal injury was significantly high $(7.2 \%)$ among hypertensive patients receiving antithrombotic drugs $(p<0.001)$. The AOR of gastrointestinal injury risk $(95 \% \mathrm{CI})$ was $1.56(1.07-2.36)$ for hypertensive patients. In general, hypertensive patients have a resistance to gastric ulcers since the gastric mucosal blood flow (a defensive factor) is well preserved in the presence of hypertension. ${ }^{8,9)}$ In treated patients with hypertension in which the blood pressure has been reduced to a goal level

Table 6. Risk Factors of Gastrointestinal Injury, Adjusted Odds Ratio by Multiple Logistic Regression Analysis

\begin{tabular}{lccr}
\hline \hline \multicolumn{1}{c}{ Risk factor } & AOR & $95 \% \mathrm{CI}$ & $p$ Value \\
\hline Hypertension & 1.56 & $1.07-2.36$ & 0.0242 \\
LDA & 1.70 & $1.17-2.50$ & 0.0061 \\
EPA & 0.62 & $0.27-1.27$ & 0.2178 \\
Clopidogrel & 2.77 & $1.70-4.49$ & $<0.0001$ \\
Warfarin potassium & 1.95 & $1.23-3.08$ & 0.0041 \\
NSAIDs & 4.13 & $2.88-5.95$ & $<0.0001$ \\
\hline AOR: adjusted odds ratio, CI: confidence interval, LDA: low-dose aspirin, EPA: \\
eicosapentaenoic acid, NSAIDs: nonsteroidal anti-inflammatory drugs.
\end{tabular}

by hypotensive treatment, gastric mucosal blood flow is lower than that in normotensive individuals. The influence of hypotensive therapy is considered to appear earlier in the gastric mucosa than in the other organs, and it has been reported that hypotensive drugs can adversely affect the gastrointestinal mucosa (through destabilizing the peripheral blood flow triggered by an autoregulatory mechanism or through excessively reducing the blood pressure) or cause gastric ulcers. ${ }^{10)}$ Hypertensive patients often receive multiple drugs because of complications by a thrombotic disease, cerebrovascular disease, cardiovascular disorder, and so on. It seems, therefore, likely that hypertensive patients are exposed to the risk of gastrointestinal injury (due to accompanying diseases), adverse reactions to multiple drug therapy, and interactions between these factors. In elderly patients, we cannot rule out the influence from factors such as Helicobacter pylori infection, history of infection, and history of gastrointestinal injury. Therefore, it is uncertain whether hypertension is a risk factor for gastrointestinal injury. More detailed studies on the relationship between hypertension and gastrointestinal injury are desirable. In elderly patients, we cannot rule out the influence from factors such as Helicobacter pylori infection, history of infection, and history of gastrointestinal injury. Therefore, it is uncertain whether hypertension is a risk factor for gastrointestinal injury. More detailed studies on the relationship between hypertension and gastrointestinal injury are desirable.

In the multivariate logistic regression analysis, the AOR $(95 \% \mathrm{CI})$ was $4.13(2.88-5.95)$ for NSAIDs, 1.70 (1.17-2.50) for low-dose aspirin, $1.95(1.23-3.08)$ for warfarin potassium, and 2.77 (1.70-4.49) for clopidogrel, allowing us to confirm that these are independent risk factors. Regarding the mechanism of NSAIDs-induced peptic ulcers or gastrointestinal bleeding, it is known that NSAIDs suppress gastric mucosal prostaglandin formation (mediated by inhibition of cyclooxygenase-1 (COX-1)), thereby reducing the resistance and defensive potential of the gastric mucosa to acid and leading to the onset of peptic ulcers or gastrointestinal bleeding. It is also known that accumulation of NSAIDs in cells directly induces mucosal injury. ${ }^{11,12)}$ Regarding the risk of low-dose aspirin for the onset of upper gastrointestinal bleeding, the AOR has been shown to be 1.6-8.2 according to metanalyses and case-control studies. $^{2,13)}$ Warfarin potassium, suggested in the present study as a risk factor for gastrointestinal injury, is less likely to injure the gastric mucosa but tends to suppress wound healing through a hemorrhagic tendency and to stimulate the hemorrhagic tendency through long-term use or interactions with

Table 7. Relationship between Risk Factors of Gastrointestinal Injury and Age

\begin{tabular}{|c|c|c|c|c|c|c|}
\hline \multirow{2}{*}{ Age range (years) } & \multirow{2}{*}{ Cases/Controls } & \multicolumn{5}{|c|}{ AOR $95 \% \mathrm{CI} p$ value } \\
\hline & & Hypertension & LDA & Clopidogrel & Warfarin potassium & NSAIDs \\
\hline \multirow[t]{3}{*}{$50-59$} & $17 / 70$ & 1.16 & 1.73 & 2.56 & 2.10 & 6.42 \\
\hline & & $0.34-4.19$ & $0.52-6.16$ & $0.61-10.64$ & $0.34-11.76$ & $2.04-22.62$ \\
\hline & & 0.8100 & 0.3754 & 0.1891 & 0.3979 & 0.0021 \\
\hline \multirow[t]{3}{*}{$60-69$} & $39 / 156$ & 0.82 & 1.29 & 4.41 & 1.80 & 4.01 \\
\hline & & $0.35-1.91$ & $0.56-2.96$ & $1.56-12.43$ & $0.74-4.26$ & $1.83-8.86$ \\
\hline & & 0.6348 & 0.5457 & 0.0046 & 0.1871 & 0.0005 \\
\hline \multirow[t]{3}{*}{$\geq 70$} & $102 / 408$ & 2.02 & 1.91 & 3.07 & 2.45 & 4.26 \\
\hline & & $1.19-3.56$ & $1.17-3.16$ & $1.62-5.77$ & $1.35-4.43$ & $2.65-6.93$ \\
\hline & & 0.0116 & 0.0103 & 0.0005 & 0.0030 & $<0.0001$ \\
\hline
\end{tabular}

LDA: low-dose aspirin, AOR: adjusted odds ratio, CI: confidence interval, NSAIDs: nonsteroidal anti-inflammatory drugs. 
other drugs, allowing us to estimate that warfarin potassium is involved in delayed healing or aggravation of existing gastrointestinal lesions. ${ }^{14)}$

Clopidogrel is not a primary cause of gastroduodenal ulcers but it is considered as one of the factors responsible for gastrointestinal injury because it inhibits vascularization and, thus, suppresses the healing of existing ulcers and erosions. ${ }^{15,16)}$ According to an overseas case-control study, the AOR for the risk of upper gastrointestinal bleeding was 2.3 (0.9-6.0) for clopidogrel; however, this ratio was not statistically significant. ${ }^{17)}$ In a study involving follow-up of patients for 1 year after the start of clopidogrel treatment, clopidogrel was shown to serve as a significant risk factor in patients with a history of upper gastrointestinal bleeding, with an AOR of $1.3(1.1-1.5){ }^{18)}$ Thus, there is no consistent finding about the risk of clopidogrel treatment for the onset of hemorrhagic gastrointestinal injury. ${ }^{14,17)}$ Although the effectiveness of PPIs as a means of preventing and treating gastrointestinal ulcers caused by low-dose aspirin has been demonstrated, no drug therapy to address the suggested risk of clopidogrel-induced gastrointestinal injury has been established. Although the magnitude of this risk varies among reports, it seems essential to explore drugs capable of reducing or preventing gastrointestinal injury and to evaluate the usefulness of concomitant use of such drugs so that clopidogrel can be used safely and continuously.

When the influence of age was analyzed, it was shown that elderly patients (aged 70 years or older) are more likely to develop gastrointestinal injury following treatment with lowdose aspirin, clopidogrel, warfarin potassium, or NSAIDs. In general, the prevalence of peptic ulcers has been shown to have an upward tendency in elderly people. Possible reasons for this tendency are as follows: (1) higher detection rate at early stages in recent years, (2) high prevalence of accompanying diseases that are likely to cause blood flow disturbance in elderly people, and (3) high number of drugs that can cause ulcers. In the present study, NSAIDs were identified as a risk factor of gastrointestinal injury in each age group over 50 years, suggesting the necessity of avoiding the use of NSAIDs without evident necessity, regardless of the age group to which a given patient belongs.

The results from this study suggest the possibility that EPA has a safety profile that is completely different from that of the other antithrombotic drugs. Although the AOR for EPA was not statistically significant, its unadjusted odds ratio $(95 \%$ CI) was $0.43(0.20-0.84)$, which indicates a significantly lower risk of gastrointestinal injury. In this study, we confirmed that the number of patients concurrently prescribed with EPA and gastric agents were few; 9 patients received PPIs (10.3\%) and 15 patients received H2ARs $(17.2 \%)$ in EPA group $(n=87)$. Thus, other factors may be considered. This is probably attributable to the following factors: (1) EPA promotes the repair of gastric epithelial cells in a dose-dependent manner; however, this is an in vitro finding ${ }^{19)}$ and (2) EPA prevents gastric ulcers through reinforcing the host defensive factors mediated by the inhibition of factors that damage the mucosa and oxidative stress. $^{20)}$ It has been reported that when EPA was administered for 2 months or longer to children with Crohn's disease, the erythrocyte EPA level increased and the leukotriene B4 level (a factor with powerful inflammatory activity, produced in leukocytes and intestinal mucosa) decreased. ${ }^{21)}$ Therefore, prolonged use of EPA can reduce the risk of gastrointestinal injury through its antiulcer or anti-inflammatory action on the digestive tract.

According to the current "Guidelines on the Use of Anticoagulants and Antiplatelet Agents for Cardiovascular Diseases" (revised version, 2009), EPA treatment is an Evidence Class 1 measure to manage high-risk dyslipidemia in patients at elevated risk of cardiovascular disease. Increasing numbers of reports on EPA are available, allowing a more reliable evaluation of this drug.) In addition, EPA has neutral fat-lowering and anti-inflammatory properties. In the Japan EPA lipid intervention study (JELIS study), EPA was shown to prevent thrombosis to an extent comparable to that achieved by aspirin, although a direct comparison between these 2 drugs was not carried out. ${ }^{22)}$ More recently, addition of $\omega 3$-polyvalentsaturated fatty acid to the standard therapy (low-dose aspirin+clopidogrel) for patients after percutaneous coronary intervention was reported to augment the antiplatelet response remarkably. ${ }^{23}$ Furthermore, EPA rarely induced adverse events and has been proven to have a favorable safety profile; its usefulness in clopidogrel resistance has also been demonstrated. ${ }^{23}$ ) These findings suggest that if EPA is added to an antiplatelet drug therapy, antiplatelet activity can be obtained without increasing the hemorrhagic risk.

In conclusion, this study confirmed that clopidgrel, warfarin and low dose aspirin are relevant risk factors for gastrointestinal injury among the antithrombotic drugs currently available. Moreover, the present study suggested for the first time that EPA treatment provides a safe means of suppressing or preventing gastrointestinal injury. Although, this study, designed as a retrospective study, has many limitations, the results from this study will be useful for the future use of antithrombotic drugs and NSAIDs, including their use in elderly patients, as antithrombotic therapy with clopidogrel, and so on.

\section{REFERENCES}

1) The Japanese Circulation Society. "Guidelines for management of anticoagulant and antiplatelet therapy in cardiovascular disease (JCS 2009).”: 〈http://www.j-circ.or.jp/english/index.html〉, cited 15 April, 2011.

2) Sakamoto C, Sugano K, Ota S, Sakaki N, Takahashi S, Yoshida Y, Tsukui T, Osawa H, Sakurai Y, Yoshino J, Mizokami Y, Mine T, Arakawa T, Kuwayama H, Saigenji K, Yakabi K, Chiba T, Shimosegawa T, Sheehan JE, Perez-Gutthann S, Yamaguchi T, Kaufman DW, Sato T, Kubota K, Terano A. Case-control study on the association of upper gastrointestinal bleeding and nonsteroidal antiinflammatory drugs in Japan. Eur. J. Clin. Pharmacol., 62, 765-772 (2006).

3) Tamura I, Fujita T, Tsumura H, Morita Y, Yoshida M, Toyonaga T, Hirano S, Inokuchi H, Kutsumi H, Azuma T. Low-dose aspirininduced gastroduodenal mucosal injury in Japanese patients with arteriosclerotic disease. Intern. Med., 49, 2537-2545 (2010).

4) Yamamoto T, Sanaka M, Nagasawa K, Abe K, Fukami M, Nakayama S, Tsuchiya A, Ishii T, Kuyama Y. Gastroduodenal mucosal injury in patients on antiplatelet therapy. Thromb. Res., 120, 465469 (2007).

5) Yamamoto T, Isono A, Ebato T, Mishina Y, Shirasaki Y, Abe K, Hattori K, Ishii T, Kuyama Y. Prevalence of gastroduodenal mucosal injury in asymptomatic patients taking antiplatelet agents in Japan. Int. J. Clin. Pharmacol. Ther., 47, 722-725 (2009).

6) Mehta SR, Yusuf S, Peters RJ, Bertrand ME, Lewis BS, Natarajan MK, Malmberg K, Rupprecht H, Zhao F, Chrolavicius S, Copland 
I, Fox KA. Clopidogrel in Unstable angina to prevent Recurrent Events trial (CURE) Investigators. Effects of pretreatment with clopidogrel and aspirin followed by long-term therapy in patients undergoing percutaneous coronary intervention: the PCI-CURE study. Lancet, 358, 527-533 (2001).

7) Yusuf S, Zhao F, Mehta SR, Chrolavicius S, Tognoni G, Fox KK, Clopidogrel in Unstable Angina to Prevent Recurrent Events Trial Investigators. Effects of clopidogrel in addition to aspirin in patients with acute coronary syndromes without ST-segment elevation. $N$. Engl. J. Med., 345, 494-502 (2001).

8) Medalie JH, Neufeld HN, Goldbourt U, Kahn HA, Riss E, Oron D. Association between blood-pressure and peptic-ulcer incidece. Lancet, 2, 1225-1226 (1970).

9) Glass GB. Blood pressure and peptic ulcer. Lancet, 1, 864 (1971).

10) Mizuno T, Shindo K. Acute gastric ulceration due to excessive reduction of blood pressure in hypertensive patients. Jpn. J. Med., 28, 269-271 (1989).

11) Robert A, Nezamis JE, Lancaster C, Hanchar AJ. Cytoprotection by prostaglandins in rats. Prevention of gastric necrosis produced by alcohol, $\mathrm{HCl}, \mathrm{NaOH}$, hypertonic $\mathrm{NaCl}$, and thermal injury. Gastroenterology, 77, 433-443 (1979).

12) Takahashi M, Ota S, Hata Y, Mikami Y, Azuma N, Nakamura T, Terano A, Omata M. Hepatocyte growth factor as a key to modulate anti-ulcer action of prostaglandins in stomach. J. Clin. Invest., 98, 2604-2611 (1996).

13) Derry S, Loke YK. Risk of gastrointestinal haemorrhage with long term use of aspirin: meta-analysis. BMJ, 321, 1183-1187 (2000).

14) Hallas J, Dall M, Andries A, Andersen BS, Aalykke C, Hansen JM, Andersen M, Lassen AT. Use of single and combined antithrombotic therapy and risk of serious upper gastrointestinal bleeding: population based case-control study. BMJ, 333, 726-728 (2006).

15) Abraham NS, Hlatky MA, Antman EM, Bhatt DL, Bjorkman DJ, Clark CB, Furberg CD, Johnson DA, Kahi CJ, Laine L, Mahaffey KW, Quigley EM, Scheiman J, Sperling LS, Tomaselli GF, ACCF/ ACG/AHA. ACCF/ACG/AHA 2010 expert consensus document on the concomitant use of proton pump inhibitors and thienopyridines: a focused update of the ACCF/ACG/AHA 2008 expert consensus document on reducing the gastrointestinal risks of antiplatelet therapy and NSAID use. A Report of the American College of Cardiology Foundation Task Force on Expert Consensus Documents. J. Am. Coll. Cardiol., 56, 2051-2066 (2010).
16) Abraham NS, Hlatky MA, Antman EM, Bhatt DL, Bjorkman DJ, Clark CB, Furberg CD, Johnson DA, Kahi CJ, Laine L, Mahaffey KW, Quigley EM, Scheiman J, Sperling LS, Tomaselli GF, ACCF/ ACG/AHA. ACCF/ACG/AHA 2010 expert consensus document on the concomitant use of proton pump inhibitors and thienopyridines: a focused update of the ACCF/ACG/AHA 2008 expert consensus document on reducing the gastrointestinal risks of antiplatelet therapy and NSAID use. Am. J. Gastroenterol., 105, 2533-2549 (2010).

17) Ibáñez L, Vidal X, Vendrell L, Moretti U, Laporte JR. SpanishItalian Collaborative Group for the Epidemiology of Gastrointestinal Bleeding. Upper gastrointestinal bleeding associated with antiplatelet drugs. Aliment. Pharmacol. Ther., 23, 235-242 (2006).

18) Ng FH, Wong SY, Chang CM, Chen WH, Kng C, Lanas AI, Wong BC. High incidence of clopidogrel-associated gastrointestinal bleeding in patients with previous peptic ulcer disease. Aliment. Pharmacol. Ther., 18, 443-449 (2003).

19) Takada H, Takahashi M, Kuwayama H. Effect of eicosapentaenoic acid (EPA) on gastric epithelial cell restitution is mediated by superoxide scavenge action. Ulcer Research, 29, 33-36 (2002).

20) Bhattacharya A, Ghosal S, Bhattacharya SK. Effect of fish oil on offensive and defensive factors in gastric ulceration in rats. Prostaglandins Leukot. Essent. Fatty Acids, 74, 109-116 (2006).

21) Shimizu T, Fujii T, Suzuki R, Igarashi J, Ohtsuka Y, Nagata S, Yamashiro Y. Effects of highly purified eicosapentaenoic acid on erythrocyte fatty acid composition and leukocyte and colonic mucosa leukotriene B4 production in children with ulcerative colitis. $J$. Pediatr. Gastroenterol. Nutr., 37, 581-585 (2003).

22) Yokoyama M, Origasa H, Matsuzaki M, Matsuzawa Y, Saito Y, Ishikawa Y, Oikawa S, Sasaki J, Hishida H, Itakura H, Kita T, Kitabatake A, Nakaya N, Sakata T, Shimada K, Shirato K, Japan EPA lipid intervention study (JELIS) Investigators. Effects of eicosapentaenoic acid on major coronary events in hypercholesterolaemic patients (JELIS): a randomised open-label, blinded endpoint analysis. Lancet, 369, 1090-1098 (2007).

23) Gajos G, Rostoff P, Undas A, Piwowarska W. Effects of polyunsaturated omega-3 fatty acids on responsiveness to dual antiplatelet therapy in patients undergoing percutaneous coronary intervention: the OMEGA-PCI (OMEGA-3 fatty acids after pci to modify responsiveness to dual antiplatelet therapy) study. J. Am. Coll. Cardiol., 55, 1671-1678 (2010). 